\title{
Research Article \\ Robust Speech Watermarking Procedure in the Time-Frequency Domain
}

\author{
Srdjan Stanković, Irena Orović, and Nikola Žarić \\ Electrical Engineering Department, University of Montenegro, 81000 Podgorica, Montenegro
}

Correspondence should be addressed to Irena Orović, irenao@cg.ac.yu

Received 18 January 2008; Accepted 16 April 2008

Recommended by Gloria Menegaz

\begin{abstract}
An approach to speech watermarking based on the time-frequency signal analysis is proposed. As a time-frequency representation suitable for speech analysis, the S-method is used. The time-frequency characteristics of watermark are modeled by using speech components in the selected region. The modeling procedure is based on the concept of time-varying filtering. A detector form that includes cross-terms in the Wigner distribution is proposed. Theoretical considerations are illustrated by the examples. Efficiency of the proposed procedure has been tested for several signals and under various attacks.
\end{abstract}

Copyright (C) 2008 Srdjan Stanković et al. This is an open access article distributed under the Creative Commons Attribution License, which permits unrestricted use, distribution, and reproduction in any medium, provided the original work is properly cited.

\section{INTRODUCTION}

Digital watermarking has been developed as an effective solution for multimedia data protection. Watermarking usually assumes embedding of secret signal that should be robust and imperceptible within the host data. Also, reliable watermark detection must be provided. A number of proposed watermarking techniques refer to the speech and audio signals [1]. Some of them are based on spreadspectrum method [2-4], while the others are related to the time-scale method $[5,6]$, or fragile content features combined with robust watermarking [7].

The existing watermarking techniques are mainly based on either the time or frequency domain. However, in both cases, the time-frequency characteristics of watermark do not correspond to the time-frequency characteristics of speech signal. It may cause watermark audibility, because the watermark will be present in the time-frequency regions where speech components do not exist. In this paper, a time-frequency-based approach for speech watermarking is proposed. The watermark in the time-frequency domain is modeled to follow specific speech components in the selected time-frequency regions. Additionally, in order to provide its imperceptibility, the energy of watermark is adjusted to the energy of speech components. In image watermarking, an approach based on the two-dimensional space/spatial frequency distribution has already been proposed in [8]. However, it is not appropriate in the case of speech signals.

Among all time-frequency representations, the spectrogram is the simplest one. However, it has a low timefrequency resolution. On the other hand, the Wigner distribution, as one of the commonly used, produces a large amount of cross-terms in the case of multicomponent signals. Thus, the S-method, as a cross-terms free timefrequency representation, can be used for speech analysis. The watermark is created by modeling time-frequency characteristics of a pseudorandom sequence according to the certain time-frequency speech components. The main problem in these applications is the inversion of the timefrequency distributions. A procedure based on the timevarying filtering has been proposed in [9]. The Wigner distribution has been used to create time-varying filter that identifies the support of a monocomponent chirp signal. However, it cannot be used in the case of multicomponent speech signals. Also, some interesting approaches to signal's components extraction from the time-frequency plane have been proposed in $[10,11]$.

In this work, the time-varying filtering, based on the cross-terms free time-frequency representation, is adapted for speech signals and watermarking purpose. Namely, this concept is used to identify the support of certain speech components in the time-frequency domain and to model the 
watermark according to these components. The basic idea of this approach has been introduced in [12]. The time-varying filtering is also used to overcome the problem of inverse mapping from the time-frequency domain. Additionally, a reliable procedure for blind watermark detection is provided by modifying the correlation detector in the time-frequency domain. It is based on the Wigner distribution, because the presence of cross-terms improves detection results [13]. Therefore, the main advantage of the proposed method is in providing efficient watermark detection with low probabilities of error for a set of strong attacks. Payload provided by this procedure is suitable for various applications [1].

The paper is organized as follows. Time-frequency representations and the concept of time-varying filtering are presented in Section 2. A proposal for watermark embedding and detection is given in Section 3. The evaluation of the proposed procedure is performed by the various examples and tests in Section 4. Concluding remarks are given in Section 5.

\section{THEORETICAL BACKGROUND}

Time-frequency representations of speech signal and the concept of time-varying filtering will be considered in this Section.

\subsection{Time-frequency representation of speech signals}

Time-frequency representations have been used for speech signal analysis. The Wigner distribution, as one of the commonly used time-frequency representations, in its pseudoform is defined as

$$
\begin{aligned}
\mathrm{WD}(n, k)=2 & \sum_{m=-N / 2}^{N / 2} w(m) w^{*}(-m) f(n+m) \\
& \times f^{*}(n-m) e^{-j 2 \pi 2 m k / N},
\end{aligned}
$$

where $f$ represents a signal (* denotes the conjugated function), $w$ is the window function, $N$ is the window length, while $n$ and $k$ are discrete time and frequency variables, respectively. However, if we represent a multicomponent signal (such as speech) as a sum of $M$ components $f_{i}(n)$, that is, $f(n)=\sum_{i=1}^{M} f_{i}(n)$, its Wigner distribution produces a large amount of cross-terms:

$$
\mathrm{WD}_{f}(n, k)=\sum_{i=1}^{M} \mathrm{WD}_{f}^{i}(n, k)+2 \operatorname{Real}\left\{\sum_{i=1}^{M} \sum_{j>i}^{M} \mathrm{WD}_{f}^{i j}(n, k)\right\},
$$

where $\mathrm{WD}_{f}^{i}(n, k)$ are the autoterms, while $\mathrm{WD}_{f}^{i j}(n, k)$, for $i \neq j$, represent the cross-terms. In order to preserve autoterms concentration as in the Wigner distribution, and to reduce the presence of cross-terms, the S-method (SM) has been introduced [14]:

$$
\operatorname{SM}(n, k)=\sum_{l=-L}^{L} P(l) \operatorname{STFT}(n, k+l) \operatorname{STFT}^{*}(n, k-l),
$$

where $P(l)$ is a finite frequency domain window with length $2 L+1$, while STFT is the short-time Fourier transform defined as $\operatorname{STFT}(n, k)=\sum_{m=-N / 2}^{N / 2} w(m) f(n+m) e^{-j 2 \pi m k / N}$, with window function $w(m)$. Thus, the SM of the multicomponent signal, whose components do not overlap in the time-frequency plane, represents the cross-terms free Wigner distribution of the individual signal components. By taking the rectangular window $P(l)$, the discrete form of SM can be written as

$$
\begin{aligned}
\operatorname{SM}(n, k)=\mid & \left.\operatorname{STFT}(n, k)\right|^{2} \\
& +2 \operatorname{Real}\left\{\sum_{l=1}^{L} \operatorname{STFT}(n, k+l) \operatorname{STFT}^{*}(n, k-l)\right\} .
\end{aligned}
$$

Note that the terms in summation improve the quality of spectrogram (square module of the short-time Fourier transform) toward the quality of the Wigner distribution.

The window $P(l)$ should be wide enough to enable the complete summation over the autoterms. At the same time, to remove the cross-terms, it should be narrower than the distance between the autoterms. The convergence within $P(l)$ is very fast, so that high autoterms concentration is obtained with only a few summation terms. Thus, in many applications $L<5$ can be used [14]. Unlike the Wigner distribution, the oversampling in time domain is not necessary since the aliasing components will be removed in the same way as the cross-terms. More details about the Smethod can be found in $[14,15]$.

Comparing to other quadratic time-frequency distributions, the S-method provides a significant saving in computation time. The number of complex multiplications for the $\mathrm{S}$-method is $N(3+L) / 2$, while the number of complex additions is $N(6+L) / 2[14]$ ( $N$ is the number of samples within the window $w(m))$. In the case of Wigner distribution, these numbers are significantly larger: $N(4$ $\left.+\log _{2} N\right) / 2$ for complex multiplications and $N \log _{2} 2 \mathrm{~N}$ for complex additions. It is important to note that the S-method allows simple and efficient hardware realization that has already been done [16, 17].

\subsection{Time-varying filtering}

Time-varying filtering is used in order to obtain watermark with specific time-frequency properties as well as to provide the inverse transform from the time-frequency domain. In the sequel, the general concept of the time-varying filtering is presented.

For a given signal $x$, the pseudoform of time-varying filtering, suitable for numerical realizations, has been defined as [18]

$$
H x(t)=\int_{-\infty}^{\infty} h\left(t+\frac{\tau}{2}, t-\frac{\tau}{2}\right) w(\tau) x(t+\tau) d \tau,
$$

where $w$ is a lag window, $\tau$ is a lag coordinate, while $h$ represents impulse response of the time-varying filter. Timevarying transfer function, that is, support function, has been 
defined as Weyl symbol mapping of the impulse response into the time-frequency domain [18]:

$$
L_{H}(t, \omega)=\int_{-\infty}^{\infty} h\left(t+\frac{\tau}{2}, t-\frac{\tau}{2}\right) e^{-j \omega \tau} d \tau,
$$

where $t$ and $\omega$ are time and frequency variables, respectively.

Thus, by using the support function (6), the filter output can be obtained as [18]

$$
H x(t)=\frac{1}{2 \pi} \int_{-\infty}^{\infty} L_{H}(t, \omega) \operatorname{STFT}_{x}(t, \omega) d \omega .
$$

The discrete form of the above relation can be written as

$$
H x(n)=\frac{1}{N} \sum_{k=-N / 2}^{N / 2} L_{H}(n, k) \operatorname{STFT}_{x}(n, k),
$$

where $\mathrm{STFT}_{x}$ is the STFT of an input signal $x$, while $N$ is the length of window $w(m)$. According to (8), by using the STFT of a pseudorandom sequence and a suitable support function, the watermark with specific time-frequency characteristics will be obtained [12]. The support function will be defined in the form of time-frequency mask that corresponds to certain speech components.

\section{WATERMARKING PROCEDURE USING TIME-FREQUENCY REPRESENTATION}

A method for time-frequency-based speech watermarking is proposed in this section. The watermark is embedded in the components of a voiced speech part. It is modeled to follow the time-frequency characteristics of significant speech formants. Furthermore, the procedure for watermark detection in the time-frequency domain is proposed.

\subsection{Watermark sequence generation}

In order to select the speech components for watermarking, the region $D$ in the time-frequency plane, that is, $D=$ $\left\{(t, \omega): t \in\left(t_{1}, t_{2}\right), \omega \in\left(\omega_{1}, \omega_{2}\right)\right\}$, is considered (see Figure 1). The time instances $t_{1}$ and $t_{2}$ correspond to the start and the end of voiced speech part. The voice activity detector, that is, word end-points detector [19-21], is used to select the voiced part of speech signal. The strongest formants are selected within the frequency interval $\omega \in\left(\omega_{1}, \omega_{2}\right)$.

The time-frequency characteristics of the watermark within the region $D$ can be modeled by using the support function defined as

$$
L_{M}(t, \omega)= \begin{cases}1, & \text { for }(t, \omega) \in D, \\ 0, & \text { for }(t, \omega) \notin D .\end{cases}
$$

Thus, the support function $L_{M}$ will be used to create a watermark with specific time-frequency characteristics. In order to use the strongest formants components, the energy floor $\xi$ is introduced. Thus, the function $L_{M}$ can be modified as

$$
L_{M}(t, \omega)= \begin{cases}1, & \text { for }(t, \omega) \in D, \text { and } \mathrm{SM}_{x}(t, \omega)>\xi, \\ 0, & \text { for }(t, \omega) \notin D, \text { or } \mathrm{SM}_{x}(t, \omega) \leq \xi,\end{cases}
$$

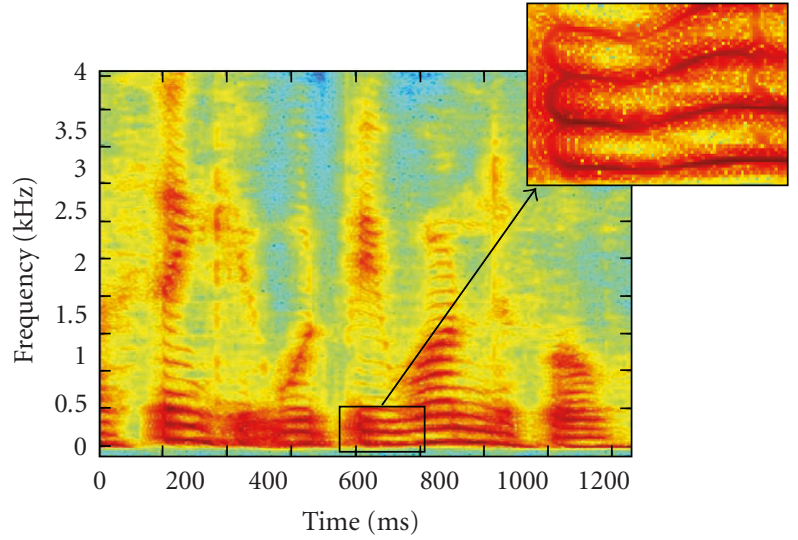

Figure 1: Illustration of the region $D$.

where $\mathrm{SM}_{x}(t, \omega)$ represents the $\mathrm{SM}$ of speech signal. Since the energy floor $\xi$ is used to avoid watermarking of weak components, an appropriate expression for $\xi$ is given by $\xi=\lambda \cdot 10^{\lambda \cdot \log _{10}\left(\max \left(\operatorname{SM}_{x}(t, \omega)\right)\right)}$, where $\max \left(\operatorname{SM}_{x}(t, \omega)\right)$ is a maximal value of signal's S-method in the region $D$, while $\lambda$ is a parameter with values between 0 and 1 . The higher $\lambda$ means that stronger components are taken. It is assumed that the significant components within the region are approximately of the same strength. It means that only a few closest formants should be considered within the region $D$. Therefore, if different time-frequency regions are used for watermarking, each energy floor should be adapted to the strength of maximal component within the considered region. It is important to note that generally, the value $\xi$ is not necessary for the detection procedure, as it will be explained latter.

The pseudorandom sequence $p$ is an input of the timevarying filter. According to (8), the watermark is obtained as

$$
w_{\text {key }}(n)=\frac{1}{N} \sum_{k=-N / 2}^{N / 2} L_{M}(n, k) \cdot \operatorname{STFT}_{p}(n, k),
$$

where $\operatorname{STFT}_{p}(n, k)$ is the discrete STFT of the sequence $p$. Since the watermark is modeled by using the function $L_{M}$, it will be present only within the specified region where the strong signal components exist.

Finally, the watermark embedding is done according to

$$
x_{w}(n)=x(n)+w_{\text {key }}(n) .
$$

\subsection{Watermark detection}

The watermark detection is performed in the time-frequency domain by using the correlation detector. The time instances $t_{1}$ and $t_{2}$ are determined by using voice activity detector. It is not necessary that the detector contains the information about the frequency range $\left(\omega_{1}, \omega_{2}\right)$ of the region $D$. Namely, the correlation can be performed along the entire frequency range of signal, but it is only effective within $\left(\omega_{1}, \omega_{2}\right)$ (region $D)$, where watermark components exist. By the way, the information about the range $\left(\omega_{1}, \omega_{2}\right)$ can be extracted from the watermark time-frequency representation. 
The detector responses must satisfy the following:

$$
\sum_{D} \operatorname{STFT}_{x_{w}}(t, \omega) \cdot \operatorname{STFT}_{w_{\mathrm{key}}}(t, \omega)>T,
$$

where $\operatorname{STFT}_{x_{w}}(t, \omega), \operatorname{STFT}_{w_{\text {key }}}(t, \omega)$ represent the short-time Fourier transform of watermarked signal and the shorttime Fourier transform of watermark, respectively, while $T$ is a threshold. The detector response for any wrong trial (sequence created in the same manner as watermark) should not be greater than the threshold value.

The support function $L_{M}$ and the energy floor $\xi$ are not required in the detection procedure. The function $L_{M}$ can be extracted from the watermark and used to model other sequences that will act as wrong trials, or simply it does not have to be used. Namely, detection can be performed even by using STFT of nonmodeled pseudorandom sequence $p$ (used to create watermark). The watermark is included in the sequence $p$, and correlation will take effect only on the time-frequency positions of watermark. The remaining parts of the sequence $p$ have the same influence on detection as in the case of wrong trials.

A significant improvement of watermark detection is obtained if the cross-terms in the time-frequency plane are included. Namely, for the calculation of SM in the detection stage, a large window length $L$ can be chosen. For the window length greater than the distance between the autoterms, cross-terms appear:

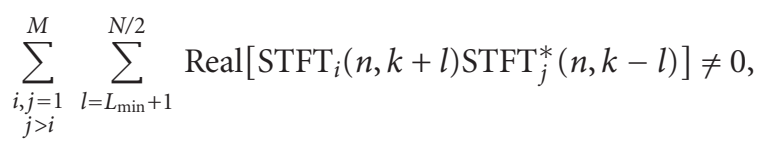

where $L_{\text {min }}$ is the minimal distance between the autoterms.

Thus, by increasing $L$ in (4), the SM approaches the Wigner distribution (for $L=N / 2$ Wigner distribution is obtained). An interesting approach to signal detection, based on the Wigner distribution, is proposed in [13], where the presence of cross-terms increases the number of components used in detection. Namely, apart from the autoterms, the watermark is included in the cross-terms as well. Therefore, by using the time-frequency domain with the cross-terms included, watermark detection can be significantly relaxed and improved, since the watermark is spread over a large number of components within the considered region. If the cross-terms are considered, the correlation detector in the time-frequency domain can be written as

$$
\text { Det }=\sum_{i=1}^{N} \mathrm{SM}_{w_{\text {key }}}^{i} \cdot \mathrm{SM}_{x_{w}}^{i}+\sum_{\substack{i, j=1 \\ i \neq j}}^{N} \mathrm{SM}_{w_{\text {key }}}^{i, j} \cdot \mathrm{SM}_{x_{w}}^{i, j}
$$

where the first summation includes autoterms, while the second one includes cross terms.

Since the cross-terms contribute in watermark detection, they should be included in other existing detectors structures. For example, the locally optimal detector based on the generalized Gaussian distribution of the watermarked coefficients, in the presence of cross terms in the timefrequency domain, can be written as

$$
\begin{aligned}
\operatorname{Det}= & \sum_{i=1}^{N} \operatorname{SM}_{w_{\mathrm{key}}}^{i} \operatorname{sgn}\left(\mathrm{SM}_{x_{w}}^{i}\right) \mid \mathrm{SM}_{x_{w}}^{i} \beta^{\beta-1} \\
& +\sum_{\substack{i, j=1 \\
i \neq j}}^{N} \operatorname{SM}_{w_{\mathrm{key}}}^{i, j} \operatorname{sgn}\left(\mathrm{SM}_{\chi_{w}}^{i, j}\right)\left|\mathrm{SM}_{\chi_{w}}^{i, j}\right|^{\beta-1} .
\end{aligned}
$$

The performance of the proposed detector is tested by using the following measure of detection quality $[22,23]$

$$
R=\frac{\bar{D}_{w_{r}}-\bar{D}_{w_{w}}}{\sqrt{\sigma_{w_{r}}^{2}+\sigma_{w_{w}}^{2}}}
$$

where $\bar{D}$ and $\sigma^{2}$ represent the mean value and the standard deviation of the detector responses, respectively, while indexes $w_{r}$ and $w_{w}$ indicate the right and wrong keys (trials). The watermarking procedure has been done for different right keys (watermarks). For each of the right keys, a certain number of wrong trials are generated in the same manner as right keys.

The probability of error $P_{\text {err }}$ is calculated by using

$$
P_{\text {err }}=p_{D w_{w}} \int_{T}^{\infty} P_{D w_{w}}(x) d x+p_{D w_{r}} \int_{-\infty}^{T} P_{D w_{r}}(x) d x,
$$

where the indexes $w_{r}$ and $w_{w}$ have the same meaning as in the previous relation, $T$ is a threshold, while equal priors $p_{D w_{w}}=$ $p_{D w_{r}}=1 / 2$ are assumed. By considering normal distribution for $P_{D w_{w}}$ and $P_{D w_{r}}$ and $\sigma_{w_{r}}^{2}=\sigma_{w_{w}}^{2}$, the minimization of $P_{\text {err }}$ leads to the following relation:

$$
P_{\text {err }}=\frac{1}{4} \operatorname{erfc}\left(\frac{R}{2}\right)-\frac{1}{4} \operatorname{erfc}\left(-\frac{R}{2}\right)+\frac{1}{2} .
$$

By increasing the value of $R$, the probability of error decreases. For example, $P_{\text {err }}(R=2)=0.0896, P_{\text {err }}(R=3)=$ 0.0169 , while $P_{\text {err }}(R=4)=0.0023$.

\section{EXAMPLES}

Efficiency of the proposed procedure is demonstrated on several examples, where signals with various maximal frequencies and signal to noise ratios (SNRs) are used. The successful detection in the time-frequency domain is performed in the case without attack as well as with a set of strong attacks.

Example 1. The speech signal with $f_{\max }=4 \mathrm{kHz}$ is considered. This maximal frequency is used to provide an appropriate illustration of the proposed method. The STFT was calculated by using rectangular window with 256 samples for time-varying filtering. Zero padding up to 1024 samples was carried out, and the parameter $L=5$ is used in the $\mathrm{SM}$ calculation. The region $D$ (Figure 2(a)) is selected to cover the first three low-frequency formants of voiced speech part. The corresponding support function $L_{M}$ (Figure 2(b)) is created by using the value $\xi$ with parameter $\lambda=0.7$. 


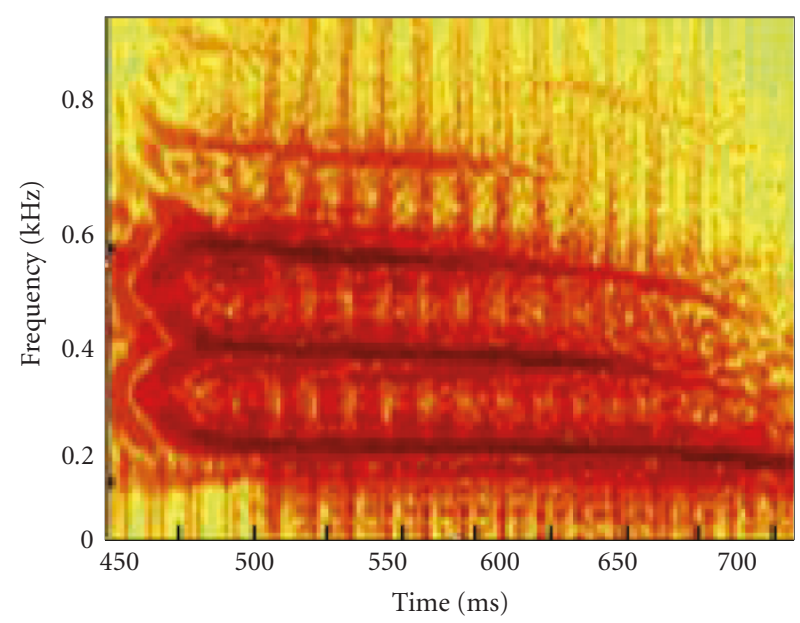

(a)

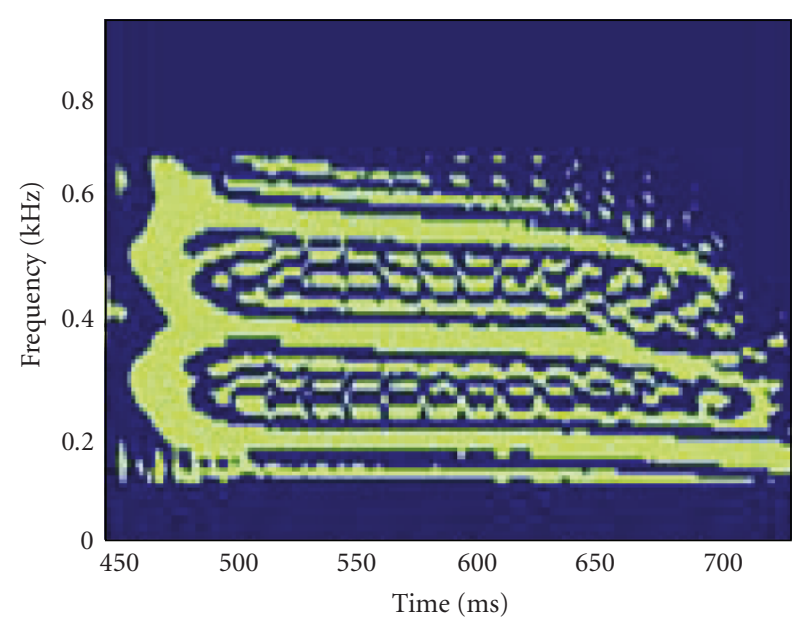

(b)

Figure 2: (a) Region $D$ of analyzed speech signal, (b) support function.

Selection of the voiced speech part is done by using the word end-points detector based on the combined Teager energy and energy-entropy features $[20,21]$ (a nonoverlapping speech frames of length 8 milliseconds are used). The original and watermarked signals are given in Figure 3(a).

The obtained SNR is higher than $20 \mathrm{~dB}$, which fulfills the constraint of watermark imperceptibility [24]. The watermark imperceptibility has also been proven by using the ABX listening test, where A, B, and X are original, watermarked, and original or watermarked signal, respectively. The listener listens to $\mathrm{A}$ and $\mathrm{B}$. Then, listener listens to $\mathrm{X}$ and decides whether $\mathrm{X}$ is $\mathrm{A}$ or $\mathrm{B}$. Since A, B, and $\mathrm{X}$ are few seconds long, the entire signals are listened to, not only isolated segments. Three female and seven male listeners with normal hearing participated in the listening test. The test was performed few times, and from the obtained statistics it was concluded that the listeners cannot positively distinguish between watermarked and original signals.
In order to illustrate the efficiency of the proposed detector form, an isolated watermarked speech part is considered. However, it is not limited to this particular speech part but, depending on the required data payload, various voiced speech parts can be used to embed and detect watermark. Detection is performed by using 100 trials with wrong keys. The responses of the standard correlation detector for STFT coefficients are given in Figure 3(b), while the responses of the detector defined by (15) are shown in Figures 3(c) and 3(d) (for window length $L=10$ and $L=$ 32, resp.). The detector response for right key is normalized to the value 1, while the responses for wrong keys are proportionally presented.

Observe that for the same right key and the same set of wrong trials, the improvement of detection results is achieved by increasing parameter $L$ (see Figure 3 ). Thus, it is obvious that the detector performance increases with the number of cross terms. In the following experiments, $L=32$ has been used to provide reliable detection. Further increasing of $L$ does not improve results significantly. Note that a window width $N+1$ (for $L=N / 2$ ), like in the Wigner distribution, can cause the presence of cross-terms that do not contain watermark, since they could result from two nonwatermarked autoterms. These cross-terms are not desirable in watermark detection procedure.

Additionally, we have performed experiments with few other speech signals. For each signal, the low-frequency formants are used, and the watermark has been embedded with approximately the same SNR (around $24 \mathrm{~dB}$ ). The detection is performed by using (15) with $L=32$. We present the results for three of them in Figure 4 . Note that the obtained results are very similar to the ones in Figure 3(d). Thus, the detection performance is insensitive to different signals tested under same conditions.

Example 2. In the previous example, the low-frequency formants have been considered. However, different frequency regions can be used. Thus, the procedure is also tested for watermark modeled according to the middle-frequency formants. The detection results are given in Figure 5(a) ( $f_{\max }=4 \mathrm{kHz}$ and $L=32$ ). The ratio between detector responses for right key and wrong trials is lower than in the previous example, with low-frequency formants, but still satisfactory. The obtained SNR is $28 \mathrm{~dB}$. In addition, the middle frequency formants of a signal with $f_{\max }=$ $11.025 \mathrm{kHz}$ have been considered. The results of watermark detection are given in Figure 5(b) $(L=32$, and SNR = $32 \mathrm{~dB}$ ). Extended frequency range enables more space for watermarking. Thus, it allows embedding watermark with lower strength, providing higher SNR.

Example 3 (evaluation of detection efficiency and robustness to attacks). In order to evaluate the efficiency of the proposed procedure by using the measure of detection quality defined by (17), we repeated the procedure for 50 trials (for 50 right keys-watermarks). They are modeled corresponding to the low-frequency formants. For each of the right keys, a number of 60 wrong keys (trials) are generated in the same manner as right keys. The average 

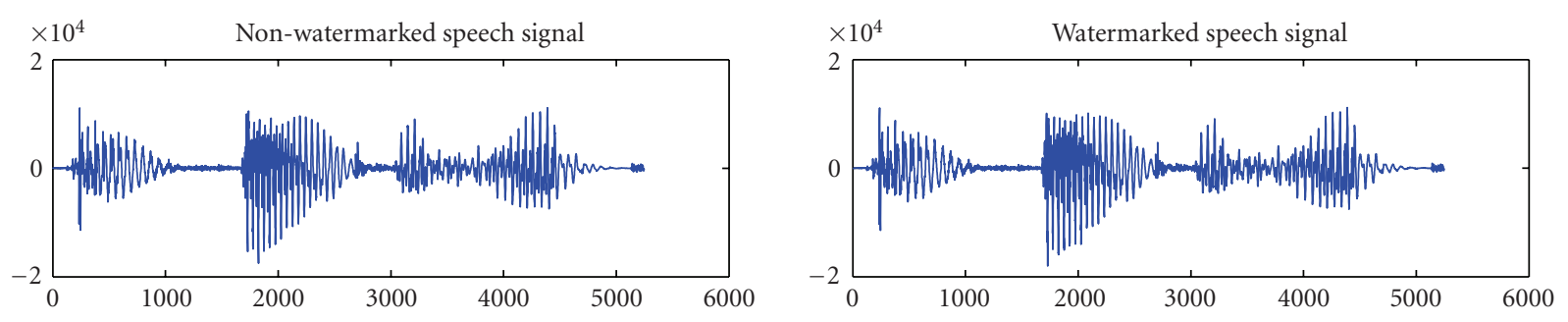

(a)
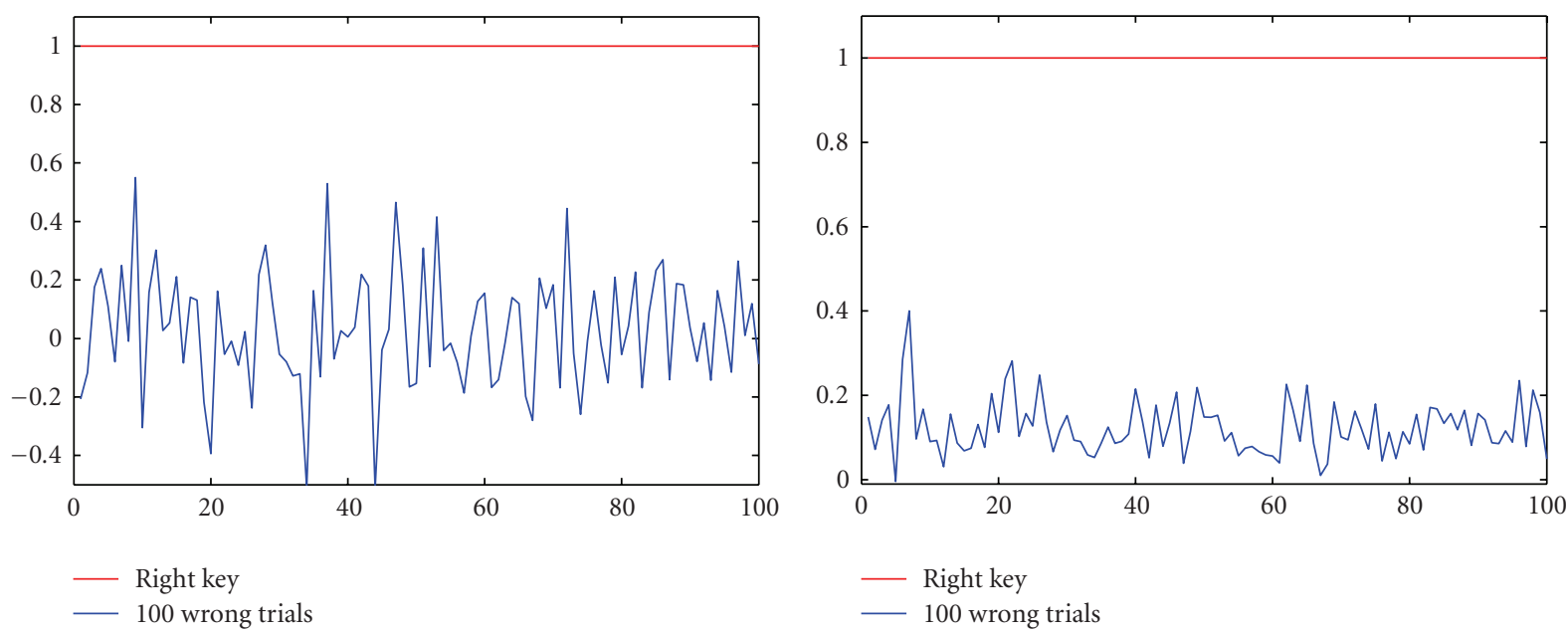

(b)

(c)

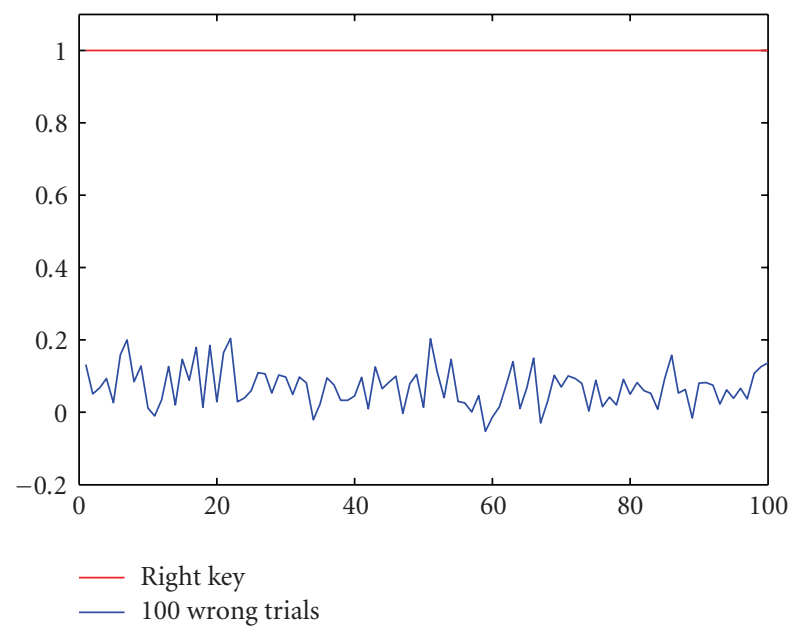

(d)

FIGURE 3: (a) Original and watermarked signals, (b) detection results for STFT coefficients, (c) detection results for SM coefficients and $L=$ 10 , (d) detection results for SM coefficients and $L=32(\mathrm{SNR}=24 \mathrm{~dB})$.

SNR is around $27 \mathrm{~dB}$. The watermark imperceptibility has been proven by using ABX listening test as in the first example. Again, the watermarked signal is perceptibly similar to the original one. The detection is performed by using correlation detector that includes cross-terms in the timefrequency domain $(L=32)$. The responses of the proposed detector for right and wrong keys are shown in Figure 6. The threshold is set as $T=\left(\bar{D}_{w_{r}}+\bar{D}_{w_{w}}\right) / 2$, where $\bar{D}_{w_{r}}$ and
$\bar{D}_{w_{w}}$ represent the mean values of the detector responses for right keys (watermarks) and wrong trials, respectively. The calculated measure of detection quality is $R=7.5$, this means that the probability of detection error is equal to $5 \cdot 10^{-8}$. The obtained probabilities of error for other signals (tested in Example 1) are of order $10^{-8}$ as well.

In the sequel, the procedure is tested on various attacks, such as Mp3 compression for different bit rates, time scaling, 


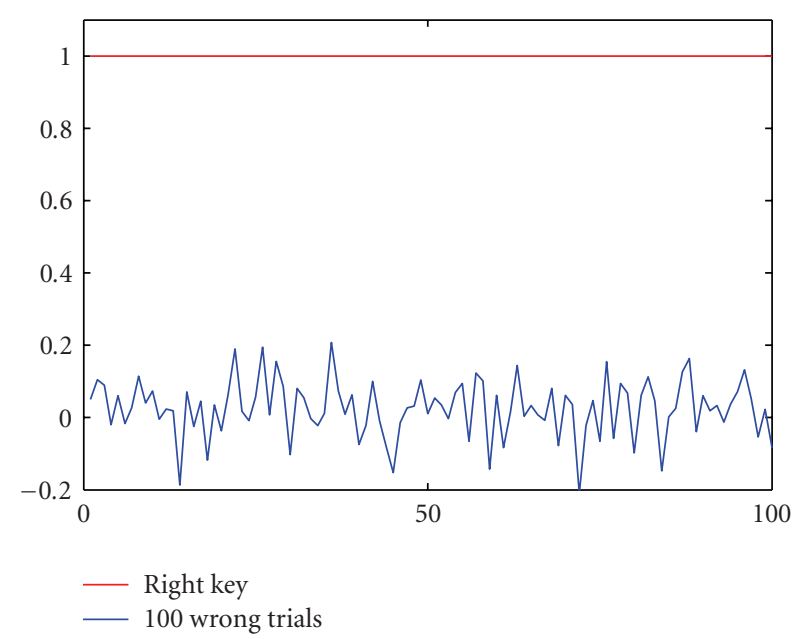

(a)

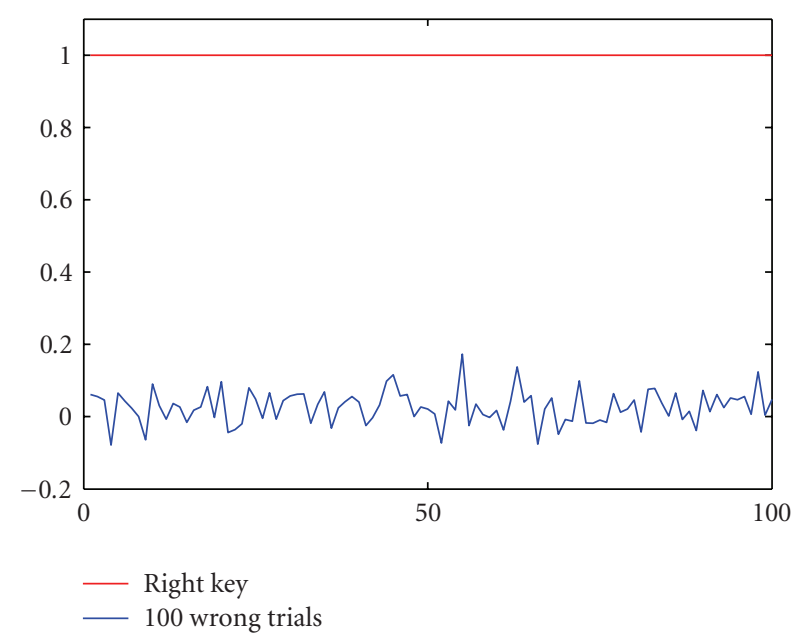

(b)

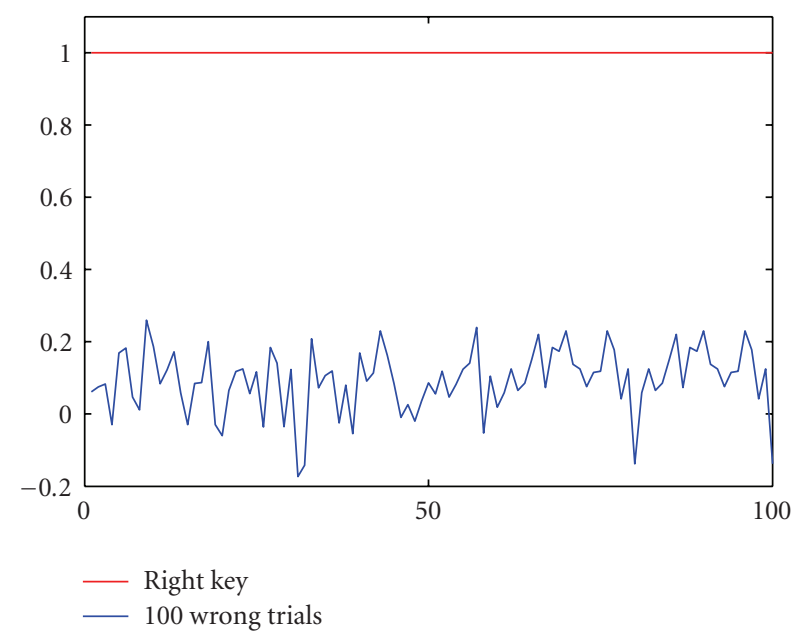

(c)

FIGURE 4: Detection results for three out of all tested signals.

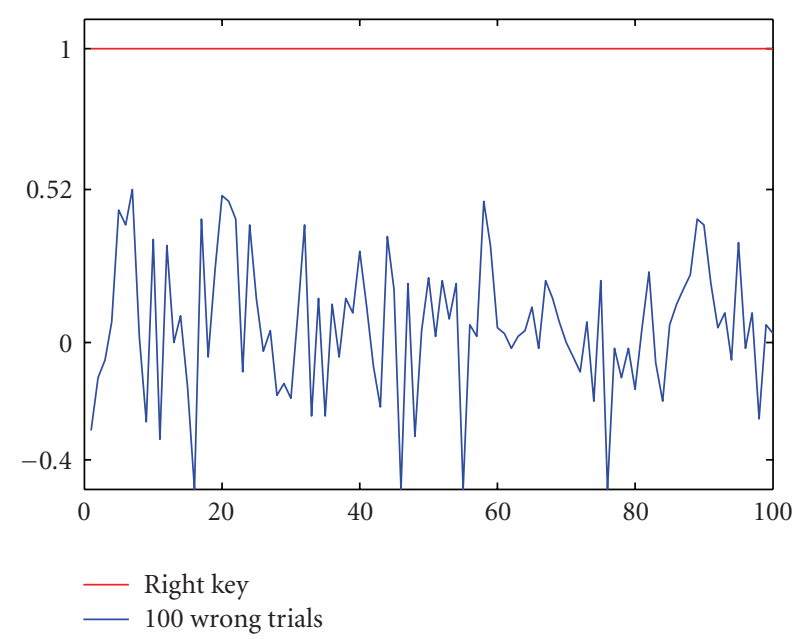

(a)

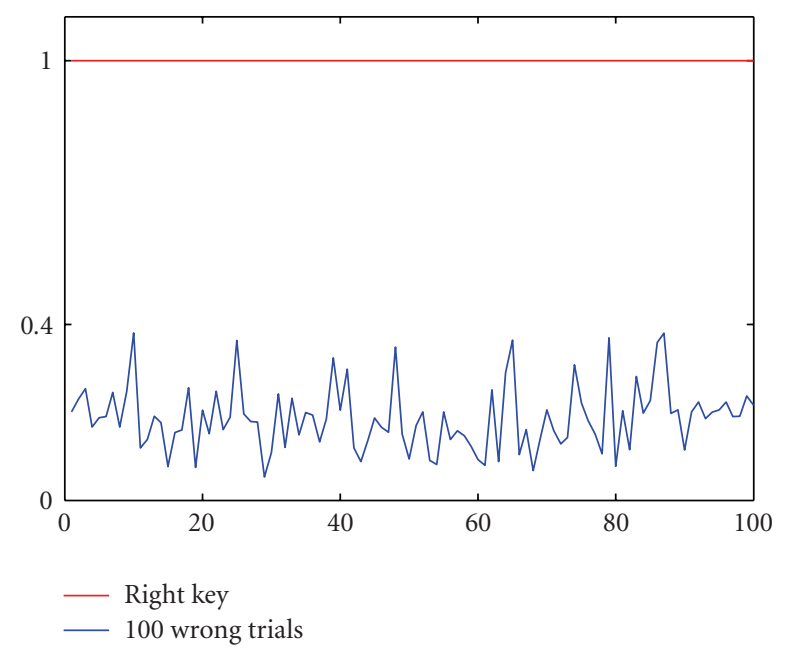

(b)

FIGURE 5: Detection results for watermark modeled to follow middle frequency formants (a) $f_{\max }=4 \mathrm{kHz}$, (b) $f_{\max }=11.025 \mathrm{kHz}$.

pitch scaling, echo, amplitudes normalization, and so forth. The results of detection in terms of quality measure $R$, and corresponding probabilities of detection error $P_{\text {err }}$ are given in Table 1. The most of attacks are realized by using CoolEditPro v2.0, while the rest of the processing is done in Matlab 7.

Note that a plenty of considered attacks are strong, and they introduce a significant signal distortion. For example, in the existing audio watermarking procedures, usually applied time scaling is up to $4 \%$, wow and flutter up to $0.5 \%$ or $0.7 \%$, echo 50 milliseconds or 100 milliseconds $[4,25]$. We have applied stronger attacks to show that, even in this case, the proposed method provides high robustness with very low probabilities of detection error (see Table 1). Note that these results were obtained with a higher watermark bit rate (more details will be provided in the 


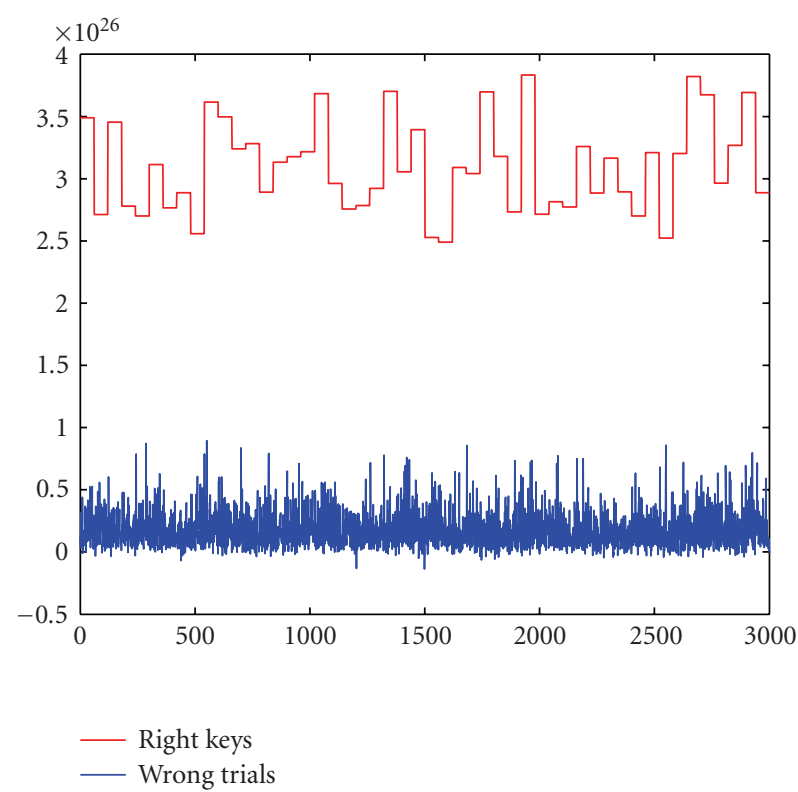

FIgURE 6: The responses of the proposed detector for 50 right keys and 3000 wrong trials.

TABLE 1: Measures of detection quality and probabilities of error for different attacks.

\begin{tabular}{lcc}
\hline Attack & $R$ & $P_{\text {err }}$ \\
\hline $\begin{array}{l}\text { No attack } \\
\text { Mp3 (constant bit rate: }\end{array}$ & 7.5 & $10^{-8}$ \\
8 Kbps) & 6.92 & $10^{-7}$ \\
$\begin{array}{l}\text { Mp3 (variable bit rate } \\
75-120 \text { Kbps) }\end{array}$ & 6.8 & $10^{-7}$ \\
$\begin{array}{l}\text { Mp3 (variable bit rate } \\
\text { 40-50 Kbps) }\end{array}$ & 6.23 & $10^{-6}$ \\
$\begin{array}{l}\text { Delay: mono light echo } \\
\text { (180 ms, mixing 20\%) }\end{array}$ & & \\
$\begin{array}{l}\text { Echo (200 ms) } \\
\text { Time stretch ( } \pm 15 \%)\end{array}$ & 6.9 & $10^{-7}$ \\
$\begin{array}{l}\text { Wow (delay 20\%) } \\
\text { Bright flutter (deep 10, }\end{array}$ & 6.8 & $10^{-7}$ \\
sweeping rate 5 Hz) & 6.2 & $10^{-6}$ \\
$\begin{array}{l}\text { Deep flutter (central freq. } \\
\text { 1000 Hz, sweeping rate 5 Hz, } \\
\text { modes-sinusoidal, filter } \\
\text { type-low pass) }\end{array}$ & 6.3 & $10^{-6}$ \\
$\begin{array}{l}\text { Amplitude: normalize (100\%) } \\
\text { Wow (delay 10\%) and bright } \\
\text { flutter }\end{array}$ & 6.8 & $10^{-7}$ \\
$\begin{array}{l}\text { Pitch scaling } \pm 5 \% \\
\text { Additive Gaussian noise (SNR } \\
=-35 \text { dB) }\end{array}$ & 6.95 & $10^{-5}$ \\
\hline & 6.82 & $10^{-7}$ \\
\hline
\end{tabular}

next subsection). The time-scale modification (TSM) is one of the challenging attacks in audio watermarking that has specially been considered in the recentliterature [24]. Very few algorithms can resist these desynchronization attacks [24]. Here, we have applied TSM-time stretch up to $\pm 15 \%$ by using software tool CoolEditPro v2.0. However, the low probability of detection error is still maintained. Only in the case of pitch scaling the obtained probability of error was lower (see Table 1), but still satisfying.

Apart from the very low probabilities of detection error, an additional advantage of the proposed detection is in providing more flexibility related to desynchronization between frequencies of the watermark sequence embedded in the signal and watermark sequence used for detection. The correlation effects are enhanced since the detection is performed within the whole time-frequency region covered with a large number of cross-terms apart from the autoterms.

In the sequel, the achieved payload and some related applications are given.

\subsection{Data payload}

In this example, we have used a single voiced part to embed a pseudorandom sequence that represents one bit of information. The approximate length of watermark, obtained as modeled pseudorandom sequences, is 1000 samples (125 milliseconds for a signal sampled at $8000 \mathrm{~Hz}$ ). Data payload varies between 4 bps and 8 bps, depending on the duration of voiced speech regions. In the case of speech signal sampled at $44100 \mathrm{~Hz}$, the achievable data payload is $22 \mathrm{bps}$. In this way we have provideda required compromise between data payload and robustness. Thus, the proposed algorithm can be efficiently used for copyright and ownership protection, copy and access control [1].

Note that the data payload can be increased by using shorter sequences. If we consider the watermark sequence with 500 samples (that correspond to 62.5 milliseconds of signal sampled at $8000 \mathrm{~Hz}$ ) the data payload is increased twice (up to $16 \mathrm{bps}$ ). However, the probability of detection error increases to $10^{-4}$. On the other hand, the probability of detection error can decrease even bellow $10^{-8}$ by considering lower watermark bit rates.

\section{CONCLUSION}

An efficient approach to watermarking of speech signals in the time-frequency domain is presented. It is based on the cross-terms free S-method and the time-varying filtering used for watermark modeling. The watermark imperceptibility is provided by adjusting the location and the strength of watermark to the selected speech components within the time-frequency region. Also, the efficient watermark detection based on the use of cross-terms in time-frequency domain is provided. The number of cross-terms employed in the detection procedure is controlled by the window length used in the calculation of S-method. The experimental results demonstrate that the procedure assures convenient and reliable watermark detection providing low probability of error. The successful watermark detection has been demonstrated in the case of various attacks. 


\section{ACKNOWLEDGMENT}

This work is supported by the Ministry of Education and Science of Montenegro.

\section{REFERENCES}

[1] N. Cvejić, Algorithms for audio watermarking and steganography, Academic Dissertation, University of Oulu, Oulu, Finland, 2004.

[2] H. J. Kim, "Audio watermarking techniques," in Proceedings of the Pacific Rim Workshop on Digital Steganography, Kyushu Institute of Technology, Kitakyushu, Japan, July 2003.

[3] I. J. Cox, J. Kilian, F. T. Leighton, and T. Shamoon, "Secure spectrum watermarking for multimedia," IEEE Transactions on Image Processing, vol. 6, no. 12, pp. 1673-1687, 1997.

[4] D. Kirovski and H. S. Malvar, "Spread-spectrum watermarking of audio signals," IEEE Transactions on Signal Processing, vol. 51, no. 4, pp. 1020-1033, 2003.

[5] C.-P. Wu, P.-C. Su, and C.-C. Jay Kuo, "Robust and efficient digital audio watermarking using audio content analysis," in Security and Watermarking of Multimedia Contents II, vol. 3971 of Proceedings of SPIE, pp. 382-392, San Jose, Calif, USA, January 2000.

[6] M. F. Mansour and A. H. Tewfik, "Audio watermarking by time-scale modification," in Proceedings of the IEEE Interntional Conference on Acoustics, Speech, and Signal Processing (ICASSP '01), vol. 3, pp. 1353-1356, Salt Lake City, Utah, USA, May 2001.

[7] M. Steinebach and J. Dittmann, "Watermarking-based digital audio data authentication," EURASIP Journal on Applied Signal Processing, vol. 2003, no. 10, pp. 1001-1015, 2003.

[8] S. Stanković, I. Djurović, and L. Pitas, "Watermarking in the space/spatial-frequency domain using two-dimensional Radon-Wigner distribution," IEEE Transactions on Image Processing, vol. 10, no. 4, pp. 650-658, 2001.

[9] S. Kay and G. Boudreaux-Bartels, "On the optimality of the Wigner distribution for detection," in Proceedings of the IEEE International Conference on Acoustics, Speech, and Signal Processing (ICASSP '85), vol. 10, pp. 1017-1020, Tampa, Fla, USA, April 1985.

[10] B. Barkat and K. Abed-Meraim, "Algorithms for blind components separation and extraction from the time-frequency distribution of their mixture," EURASIP Journal on Applied Signal Processing, vol. 2004, no. 13, pp. 2025-2033, 2004.

[11] C. Ioana, A. Jarrot, A. Quinquis, and S. Krishnan, "A watermarking method for speech signals based on the timewarping signal processing concept," in Proceedings of the IEEE International Conference on Acoustics, Speech and Signal Processing (ICASSP '07), vol. 2, pp. 201-204, Honolulu, Hawaii, USA, April 2007.

[12] S. Stanković, I. Orović, N. Žarić, and C. Ioana, "An approach to digital watermarking of speech signals in the timefrequency domain," in Proceedings of the 48th International Symposium ELMAR Focused on Multimedia Signal Processing and Communications, pp. 127-130, Zadar, Croatia, June 2006.

[13] B. Boashash, "Time-frequency signal analysis," in Advances in Spectrum Analysis and Array Processing, S. Haykin, Ed., chapter 9, pp. 418-517, Prentice Hall, Englewood Cliffs, NJ, USA, 1991.

[14] L. Stanković, "A method for time-frequency signal analysis," IEEE Transactions on Signal Processing, vol. 42, no. 1, pp. 225229, 1994.
[15] I. Djurović and L. Stanković, "A virtual instrument for timefrequency analysis," IEEE Transactions on Instrumentation and Measurement, vol. 48, no. 6, pp. 1086-1092, 1999.

[16] D. Petranović, S. Stanković, and L. Stanković, "Special purpose hardware for time frequency analysis," Electronics Letters, vol. 33, no. 6, pp. 464-466, 1997.

[17] S. Stanković, L. Stanković, V. Ivanović, and R. Stojanović, "An architecture for the VLSI design of systems for timefrequency analysis and time-varying filtering," Annales des Telecommunications, vol. 57, no. 9-10, pp. 974-995, 2002.

[18] S. Stanković, "About time-variant filtering of speech signals with time-frequency distributions for hands-free telephone systems," Signal Processing, vol. 80, no. 9, pp. 1777-1785, 2000.

[19] Q. Li, J. Zheng, A. Tsai, and Q. Zhou, "Robust endpoint detection and energy normalization for real-time speech and speaker recognition," IEEE Transactions on Speech and Audio Processing, vol. 10, no. 3, pp. 146-157, 2002.

[20] G. S. Ying, C. D. Mitchell, and L. H. Jamieson, "Endpoint detection of isolated utterances based on a modified Teager energy measurement," in Proceedings of the IEEE International Conference on Acoustics, Speech, and Signal Processing (ICASSP '93), vol. 2, pp. 732-735, Minneapolis, Minn, USA, April 1993.

[21] L. Gu and S. Zahorian, "A new robust algorithm for isolated word endpoint detection," in Proceedings of the IEEE International Conference on Acoustics, Speech, and Signal Processing (ICASSP '02), vol. 4, pp. 4161-4164, Orlando, Fla, USA, May 2002.

[22] S. Stanković, I. Djurović, R. Herpers, and L. Stanković, "An approach to optimal watermark detection," AEU International Journal of Electronics and Communications, vol. 57, no. 5, pp. 355-357, 2003.

[23] B.-S. Ko, R. Nishimura, and Y. Suzuki, "Time-spread echo method for digital audio watermarking," IEEE Transactions on Multimedia, vol. 7, no. 2, pp. 212-221, 2005.

[24] S. Xiang and J. Huang, "Histogram-based audio watermarking against time-scale modification and cropping attacks," IEEE Transactions on Multimedia, vol. 9, no. 7, pp. 1357-1372, 2007.

[25] N. Cvejić and T. Seppänen, "Improving audio watermarking scheme using psychoacoustic watermark filtering," in Proceedings of the 1st IEEE International Symposium on Signal Processing and Information Technology, pp. 169-172, Cairo, Egypt, December 2001. 